\title{
Evaluation of the first two Frontline cohorts of the Field Epidemiology Training Program in Guinea, West Africa
}

Pia D. M. MacDonald ( $\sim$ pmacdonald@rti.org )

RTI International https://orcid.org/0000-0003-1340-8905

\section{Doreen Collins}

RTI International https://orcid.org/0000-0002-7036-7700

\section{Boubacar Ibrahima Diallo}

CDC: Centers for Disease Control and Prevention

\section{Mariama Boubacar Bah}

RTI International

\section{Marlyatou Bah}

RTI International

\section{Claire J. Standley}

Georgetown University

\section{Salomon Corvil}

AFENET: African Field Epidemiology Network

Lise D. Martel

CDC: Centers for Disease Control and Prevention

\section{Research Article}

Keywords: Workforce developmen, field epidemiology

Posted Date: February 7th, 2022

DOI: https://doi.org/10.21203/rs.3.rs-1284182/v1

License: (1) (1) This work is licensed under a Creative Commons Attribution 4.0 International License. Read Full License 


\section{Abstract}

\section{Background}

The 2014-2016 Ebola virus disease outbreak in West Africa revealed weaknesses in the health systems of the three most heavily affected countries, including a shortage of public health professionals at the local level trained in surveillance and outbreak investigation. In response, the Frontline Field Epidemiology Training Program (FETP) was created by CDC in 2015 as a three-month, accelerated training program in field epidemiology that specifically targets the district level. In Guinea, the first two FETP-Frontline cohorts were held from January to May, and from June to September 2017. Here, we report the results of a crosssectional evaluation of these first two cohorts of FETP-Frontline in Guinea.

\section{Methods}

The evaluation was conducted in April 2018 and consisted of interviews with graduates, their supervisors, and directors of nearby health facilities, as well as direct observation of data reports and surveillance tools at health facilities. Interviews and site visits were conducted using standardized questionnaires and checklists. Qualitative data were coded under common themes and analyzed using descriptive statistics.

\section{Results}

The evaluation revealed a significant perception of improvement in all assessed skills by the graduates, as well as high levels of self-reported involvement in key activities related to data collection, analysis, and reporting. Supervisors highlighted improvements to systematic and quality case and summary reporting as key benefits of the FETP-Frontline program. At the health facility level, staff reported the training had resulted in improvements to information sharing and case notifications. Reported barriers included lack of transportation, available support personnel, and other resources. Graduates and supervisors both emphasized the importance of continued and additional training to solidify and retain skills.

\section{Conclusions}

The evaluation demonstrated a strongly positive perceived benefit of the FETP-Frontline training on the professional activities of graduates as well as the overall surveillance system. However, efforts are needed to ensure greater gender equity and to recruit more junior trainee candidates for future cohorts. Moreover, although improvements to the surveillance system were observed concurrent with the completion of the two cohorts, the evaluation was not designed to directly measure impact on surveillance or response functions. Combined with the rapid implementation of FETP-Frontline around the world, this suggests an opportunity to develop standardized evaluation toolkits, which could incorporate metrics that would directly assess the impact of equitable field epidemiology workforce development on countries' abilities to prevent, detect, and respond to public health threats.

\section{Background}


In 1998, the World Health Organization (WHO) Regional Office for Africa launched the Integrated Disease Surveillance and Response (IDSR) strategy to promote effective use of resources for disease surveillance and response using a district health approach. However, despite commitment from the various ministries of health, a 2008 assessment of the countries in the Economic Community of West Africa States (ECOWAS) found the successful implementation of a national IDSR strategy hindered by a lack of qualified public health personnel at all levels of the public health system.[1] Other analyses have also highlighted lack of workforce capacity as a major impediment to implementation of universal health coverage in the African region.[2]

The 2014-2016 Ebola virus disease (EVD) outbreak in West Africa originated in rural Guinea through a spillover of the virus into the human population.[3] EVD transmission then rapidly spread to Liberia and Sierra Leone, highlighting the three countries' lack of public health infrastructure and system's capacity to prevent, detect, and respond to emerging infectious disease threats [4] and meet WHO's International Health Regulations (IHR) core capacity requirements for detecting, assessing, reporting and responding to potential public health emergencies of international concern. Establishing workforce capacity to prevent, detect, and respond to outbreaks and other potential public health emergencies is a critical component of the IHR.

Since 1980, the U.S. Centers for Disease Control and Prevention (CDC) has supported the establishment and implementation of Field Epidemiology Training Programs (FETPS) in over 80 countries. Modeled on CDC's Epidemic Intelligence Service, the traditional FETP structure consists of a two-year applied, inservice training program which largely focuses on developing workforce capacity at the national level. The deficits identified during the West Africa EVD outbreak, however, emphasized the need to rapidly enhance the surveillance and outbreak investigation capacity of the public health workforce at the district level. In response, FETP-Frontline was developed in 2015 as a three-month, accelerated training program in field epidemiology that specifically targets district level public health officials. Implementation of FETPFrontline required extensive cooperation between the CDC and the Ministry of Health in each country to establish the need and feasibility of the program, conduct short implementation workshops for preliminary planning, and establish the priority of personnel to be trained. Mentors for the first cohorts were identified from neighboring countries if needed, and the countries were responsible for developing plans to ensure there would be adequate coverage of FETP graduates by the end of the program. To minimize the impact of the training on the health system, classroom activities were limited to 5-day sessions at the beginning, middle, and end of the program, sandwiched between extensive 5- 6 weeks stints of field training. By the end of 2016, 1,354 people from 24 countries had successfully completed the training.[5] In Guinea, the first two FETP-Frontline cohorts were held from January to May, and from June to September 2017, from which a total of 54 trainees graduated.

While there have been numerous evaluations both of national and regional full two-year FETPs, as well as the impact of the FETP training model as a tool for global health workforce capacity building,[6-10] to date there are few published accounts on the country-level benefits or impacts of FETP-Frontline.[5],[11] Here, we report the results of a cross-sectional evaluation of the first two cohorts of FETP-Frontline in 
Guinea. The objectives were to describe the characteristics of the graduates; how participation in FETPFrontline was perceived by trainees and their supervisors; how it improved the quality of their work; and assess the alignment of the program with the surveillance system.

\section{Methods}

The evaluation consisted of interviews with graduates, their supervisors[1], and directors of health facilities that had served as field sites during the graduates' training, as well as site visits for document checks of the graduates' weekly reports, reporting tools, data summaries, and the surveillance tools at the same health facilities. The methods were based on FETP-Frontline evaluations conducted by CDC in Senegal and Côte d'Ivoire in 2016 and 2017, respectively, and were adapted to the Guinean context and implemented in the field by Research Triangle International (RTI), in collaboration with the African Field Epidemiology Network (AFENET) and CDC.

\section{Sample selection}

After the completion of the second cohort, each of the country's eight regions and 38 districts was staffed with at least one FETP-Frontline graduate (Figure 1). The study population for the evaluation included all 54 graduates of the two cohorts, their current supervisors, and the director of one health facility where each graduate conducted field exercises during their training. For the health facility interviews, the team reviewed the list of health facilities where each graduate had conducted field exercises and selected one based on its proximity to the interviewee's current work site. Proximity to the graduates' current place of employment was the only criteria for health facility selection, due to transportation constraints preventing access to sites further away.

\section{Data collection}

RTI staff with previous interviewing experience and familiarity with FETP trained four teams of two interviewers each, selected from two CDC staff, one RTI headquarter staff, and five local RTI field epidemiologists. All interviewers were fluent in French. The training included instructions on how to avoid response bias, probe for more detailed information, and listen actively by restating key points made by the interviewee.

RTI and AFENET revised questionnaires from previous evaluations to remove, edit, or add questions; changed some of the language to adapt it to the Guinean context (e.g. job titles); and reduced the number of open-ended questions. Two interview teams pilot-tested the participant questionnaire with graduates from the third cohort (not included in the evaluation) and recommended minor formatting changes to the interview tool. 
The interviews were planned for April 10-20, 2018, corresponding to 11 months post-training for Cohort 1 graduates and seven months post-training for Cohort 2 . All interviews were conducted using the standardized questionnaires, covering pre-determined topic areas (Table 1). Full questionnaires are available as Additional Files.

The interviews with the graduates were conducted in person, in private, at the graduates' place of employment. Supervisors were interviewed in person where possible, or via telephone if a face to face meeting was not feasible. Interviews with the graduates lasted from one to two hours, interviews with supervisors took up to 45 minutes, and interviews at the field visit health facilities took up to 30 minutes. At the facilities, interviewers conducted document checks and visual observations of the facilities, to assess availability of key guidance documents and other tools, and to validate information provided by the trainees.

\section{Table 1}

Questionnaire Topic Areas for FETP-Frontline Evaluation, Guinea

FETP graduate questionnaire

o Demographics

- Self-assessed competency

- Data collection, analysis, and reporting practices

o Quality and content of surveillance reports (interviewer observation)

- Obstacles to implementation

o Recommendations

Supervisor questionnaire

o Changes in work habits post-training

- Recommendations for additional training

Health facility staff

questionnaire and

observational checklist
- Changes in surveillance practices post-field visit

o Obstacles to surveillance

o Reporting guidelines (case definitions, lists of immediate and mandatory notifiable diseases) posted (interviewer observed) o Reports, data trends and archives, and rumor logs accessible (interviewer observed)

\section{Team and data management}

Interviewers worked in teams of two. Interviewers alternated between interviewing and note taking between each interview and attempted to transcribe verbatim the responses to the open-ended questions. Interviews were not recorded, as the team felt that recording might inhibit respondents, and especially graduates, from speaking freely. A data manager based in Conakry entered the complete responses into an Excel spreadsheet when the interview teams returned from the field. A second data manager then reviewed all the original questionnaires and compared them to the responses recorded in the database to confirm the responses in the database were complete and accurate. 


\section{Analysis}

Descriptive statistics were summarized for each question using $S A S \AA$ Enterprise Guide ${ }^{\circledR}$ (SAS Institute Inc., Cary, USA). All interviewee responses were included when calculating percentages unless otherwise noted in the table. After the data were entered into an Excel spreadsheet, a data manager identified common themes from participant answers to open-ended questions and developed a codebook listing all the possible codes for a specific question. Coding was done manually in the original French text so as not to lose nuances of the qualitative data. $P$ values were calculated using t-tests to compare differences in responses before and after participation in FETP-Frontline. The significance level was set at 0.05 .

\section{Ethical Statement}

The study was determined to be exempt from IRB approval as primary data collection was conducted via interviews with individuals responding in their official capacity as trainees of a government program. The Ministry of Health provided permission to conduct the evaluation.

\section{Results}

\section{Interview coverage}

Between April $10-23,2018$, the team interviewed 50 (93\%) of the 54 graduates Of the four graduates not interviewed, three were away from their town of residence and could not reschedule their interviews despite multiple attempts, and one had retired three months before the study began. The team visited one health facility for each interviewed graduate (50 in total) to conduct site observations and interview staff and interviewed 37 (74\%) of the supervisors. The remaining 13 supervisors were absent due to illnesses, trainings, or other reasons; the supervisors of the four graduates who were unable to be interviewed were not contacted for interviews.

\section{Demographic Characteristics}

Table 2 summarizes the demographic and professional characteristics of the interviewed graduates. Among those interviewed, 10 (20\%) graduates were female and the mean age of the graduates was 48 years old. The most frequently reported profession was physician (68\%). On average, the graduates from the first cohort were older and had been on the job longer than the graduates from the second cohort. Job titles did not necessarily correlate to profession; the health agents, the nurse, and the physicians all had the title Head of Disease Surveillance, which was the most commonly cited title $(60 \%)$. The titles for those described as 'other' were Acting Regional Director of Health (Direction Régionale de la Santé par intérim), Chief of IHR Unit (Chef d'unité Règlement Sanitaire International) and Head of Community-Based Surveillance (Chargé de Surveillance à Base Communautaire). At the time of the interviews, all the graduates were in the same jobs they had when they participated in FETP-Frontline. 
Table 2

Demographic Characteristics of FETP-Frontline Guinea Graduates Interviewed (Cohorts 1 and 2) 
Characteristics

Cohort 1,

$\mathrm{N}=24$

n (\%)
Cohort 2,

$\mathrm{N}=26$

n (\%)
Combined,

$\mathrm{N}=50$

n (\%)

Sex

Female

Male

Age in years

\begin{tabular}{|c|c|c|c|}
\hline Mean & $53(\mathrm{NA})$ & $44(\mathrm{NA}))$ & 48 (NA) \\
\hline Mean SD & 8 (NA) & 12 (NA) & 11 (NA) \\
\hline Median & $52(\mathrm{NA})$ & 44 (NA) & 50 (NA) \\
\hline Median Range & $32-62$ (NA) & 28-65 (NA) & $28-65(\mathrm{NA})$ \\
\hline \multicolumn{4}{|l|}{ Profession } \\
\hline Health Agent & $3(13 \%)$ & $0(0 \%)$ & $3(6 \%)$ \\
\hline Epidemiologist & $2(8 \%)$ & $1(4 \%)$ & $3(6 \%)$ \\
\hline Nurse & $1(4 \%)$ & $0(0 \%)$ & $1(2 \%)$ \\
\hline Physician & $11(46 \%)$ & $23(88 \%)$ & $34(68 \%)$ \\
\hline Public Health Specialist ${ }^{1}$ & $4(17 \%)$ & $2(8 \%)$ & $6(12 \%)$ \\
\hline Did not report & $3(13 \%)$ & $0(0 \%)$ & $3(6 \%)$ \\
\hline \multicolumn{4}{|l|}{ Job Title } \\
\hline $\begin{array}{l}\text { Deputy District Head of Disease Surveillance and } \\
\text { Control }\end{array}$ & $1(4 \%)$ & $10(38 \%)$ & $11(22 \%)$ \\
\hline Other & $1(4 \%)$ & $2(8 \%)$ & $3(6 \%)$ \\
\hline Director of Planning & $3(13 \%)$ & $2(8 \%)$ & $5(10 \%)$ \\
\hline District Head of Disease Surveillance and Control & $18(75 \%)$ & $12(46 \%)$ & $30(60 \%)$ \\
\hline Did not report & $1(4 \%)$ & $0(0 \%)$ & $1(2 \%)$ \\
\hline \multicolumn{4}{|l|}{ Years at Current Position } \\
\hline$<5$ & $10(42 \%)$ & $17(65 \%)$ & $27(54 \%)$ \\
\hline $5-10$ & $5(21 \%)$ & $7(27 \%)$ & $12(24 \%)$ \\
\hline$>10$ & $9(38 \%)$ & $2(8 \%)$ & 11 (22\%) \\
\hline
\end{tabular}


$\mathrm{N}=$ total number; $\mathrm{SD}=$ standard deviation; $\mathrm{NA}=$ Not Applicable, in cases where it was not appropriate to calculate a percentage value for the data.

1 The "public health specialist" position is translated from technician supérieur de santé publique, which is a standardized position for nurses who have received additional training in public health, surveillance and epidemiology. In Guinea, the majority of public health functions are carried out by clinical professionals such as doctors or nurses, in part reflecting the relative lack of separate public health career tracks and training within the educational system.

\section{Self-Assessment of Skills Pre- and Post-Training}

The interview team asked graduates to rate their surveillance and data analysis skills on a one (novice) to five (expert) scale before and after the FETP-Frontline training. For both cohorts combined, there was a two-point increase in the mean of self-assessed level of skill in investigating outbreaks, verifying data quality, and summarizing data (standard deviation 0.72-0.75). The increase in skill level for using Excel and PowerPoint, and for describing a public health surveillance system was slightly less than two points (standard deviation 0.73-0.90). All differences were statistically significant $(p=<0.001)$. (Table 3$)$

The two cohorts did not have statistically significant differences in before and after scores, except for "verifying data quality", for which Cohort 1 cited on average a slightly smaller difference in self-perceived improvement after the training (mean difference 2.02 versus 2.21; standard deviation 0.51 versus 0.91 ; $\mathrm{p}=0.01)$.

\section{Table 3}

Self-Assessed Competency Before and After Participation in FETP Frontline: Both Cohorts $(\mathrm{N}=50)$ 


\begin{tabular}{|c|c|c|c|c|c|}
\hline \multirow[t]{2}{*}{ Skill } & $\begin{array}{l}\text { Before } \\
\text { Training }\end{array}$ & $\begin{array}{l}\text { After } \\
\text { Training }\end{array}$ & Difference & \multirow[t]{2}{*}{$\begin{array}{l}\text { Percentage } \\
\text { Improvement }\end{array}$} & \multirow[t]{2}{*}{$\begin{array}{l}P \\
\text { value }\end{array}$} \\
\hline & Mean (SD) & $\begin{array}{l}\text { Mean } \\
\text { (SD) }\end{array}$ & $\begin{array}{l}\text { Mean } \\
\text { (SD) }\end{array}$ & & \\
\hline PowerPoint & $2.47(1.05)$ & $\begin{array}{l}4.03 \\
(0.70)\end{array}$ & $\begin{array}{l}1.56 \\
(0.87)\end{array}$ & $63.2 \%$ & $<.001$ \\
\hline Excel & $2.31(0.99)$ & $\begin{array}{l}3.97 \\
(0.66)\end{array}$ & $\begin{array}{l}1.66 \\
(0.90)\end{array}$ & $71.9 \%$ & $<.001$ \\
\hline $\begin{array}{l}\text { Describing public health } \\
\text { surveillance }\end{array}$ & $2.43(0.70)$ & $\begin{array}{l}4.23 \\
(0.48)\end{array}$ & $\begin{array}{l}1.79 \\
(0.73)\end{array}$ & $73.7 \%$ & $<.001$ \\
\hline $\begin{array}{l}\text { Investigating/Responding to } \\
\text { outbreaks }\end{array}$ & $2.20(0.75)$ & $\begin{array}{l}4.20 \\
(0.46)\end{array}$ & $\begin{array}{l}2.00 \\
(0.72)\end{array}$ & $90.9 \%$ & $<.001$ \\
\hline Summarizing data & $2.15(0.66)$ & $\begin{array}{l}4.24 \\
(0.41)\end{array}$ & $\begin{array}{l}2.09 \\
(0.75)\end{array}$ & $97.2 \%$ & $<.001$ \\
\hline Verifying data quality & $2.02(0.75)$ & $\begin{array}{l}4.14 \\
(0.57)\end{array}$ & $\begin{array}{l}2.12 \\
(0.75)\end{array}$ & $105.0 \%$ & $<.001$ \\
\hline
\end{tabular}

\section{Data Collection, Analysis and Reporting Practices}

Forty-seven graduates (94\%) reported collecting data on notifiable diseases in the two months prior to the interview (Table 4). Graduates reviewed original reports with reporting health facility staff to ensure the reports were complete, and then compared the data in the reports to data in the register. If there were any discrepancies between the register and the reports, graduates worked with health facility staff to resolve them and update the data. Graduates also performed other quality assurance activities including comparing data from the previous week, following up on missing or incomplete reports, and performing supervision visits.

Thirty-one graduates (62\%) reported they had participated in an investigation in the two months prior to the interview. Forty-eight (96\%) stated that in 2017, they had the opportunity to test their newly acquired skills during a measles outbreak. During this outbreak, 38 graduates (79\%) investigated suspect cases, 21 (44\%) investigated and reported outbreaks, 25 (52\%) organized a response to the outbreak, $10(21 \%)$ started a vaccination campaign, and nine (19\%) provided community training.

Table 4

Survey of Data Collection, Analysis, and Reporting Practices 
Have you collected data on notifiable diseases over the course of the last two months?

47

$(94 \%)$

45

$(90 \%) \quad(10 \%)$

Have you conducted supervision visits at the sites that send you surveillance data over the course of the last two months?

Have you done a data quality audit over the course of the last two months?

Have you cleaned/validated surveillance data over the course of the last two months?

Have you participated in a case or outbreak investigation over the course of the last two months?

Analysis

Have you recently tabulated and analyzed surveillance data?

Are your analyses of disease trends on display at your workplace?

Tools used for data analysis:

Excel

Epi Info

Manual analysis

$34 \quad 16$

$(68 \%)$

\section{9}

(98\%)

1

31

(62\%)

19

Yes No

48

(96\%)

2

(4\%)

41

(82\%)

9

49

(98\%)

1 (2\%)

2 (4\%)

\section{Reporting}

Do you submit weekly surveillance reports to the next level in the disease surveillance system?

48

$(96 \%)$

Format of reporting for those submitting weekly reports $(\mathrm{N}=48)$ :

Email

DHIS2
2

$(4 \%)$

$\mathrm{N} / \mathrm{A}$
46

$(97 \%)$

33

$(67 \%)$

Forty $(80 \%)$ graduates provided data analysis results to the health facilities on the surveillance data they had sent. They primarily share these analyses orally at routine weekly or monthly meetings held by the health facility. Only five (10\%) graduates reported sharing the analyses by phone or email. The primary reason the graduates did not provide analysis results to the health centers by email was because the health center staff lacked computer skills. Other barriers to sharing data analyses were lack of electricity or internet connection and lack of resources to produce hard copies of the analysis results. 
All but one supervisor cited the systematic analysis of case reports or data summaries as an improvement since the graduate completed training. Figure 2 summarizes the other improvements mentioned by the supervisors. Twenty-eight (76\%) of supervisors indicated an improvement in the completeness and timeliness of the reports, as measured by reports that had no missing data and were submitted on time, whether weekly or monthly, compared to previous reports. Eighteen (49\%) said the graduates' reports included graphs and charts to summarize and interpret data, indicating improvement in quality and analysis of the data, and four $(11 \%)$ said the data had documentation of validation, compared to previous reports. All but one of the supervisors (97\%) reported that the graduates were routinely analyzing case reports and data summaries they received before submitting summary reports.

\section{Perceived Benefit to the Surveillance System}

Graduates reported that the most important outcome of their training was the improvement to data quality (Figure 3). Twelve (30\%) of graduates' supervisors mentioned an improvement in the overall coordination and collaboration throughout the system, and $11(30 \%)$ said the graduates were more motivated and engaged in surveillance activities.

In terms of perceived ability to implement measures to protect public health, 48 graduates (96\%) said their analysis of the data enables them to follow the trends of reportable disease, identify outbreaks early, initiate investigations, and use surveillance data to make recommendations to improve public health or surveillance procedures. The graduates reported that based on their recommendations, there had been a vaccination campaign, integration of the private health clinics into the surveillance system,

enhancements to the process for ensuring data quality, and additional funds or personnel. All supervisors reported that the graduates had worked to increase knowledge of case definitions in their districts, and 34 supervisors (92\%) reported that the graduates had conducted a surveillance training in the district for community-level health facilities' staff.

Forty-eight (96\%) of the health facility staff interviewed said there were positive changes in surveillance and response activities since the graduate's visit during their training. Twenty-nine interviewees $(60 \%)$ reported that information sharing and case notifications had improved. Other improvements were reported in: 1) posting of case definitions in the facility; 2) availability of resources; 3 ) data quality; 4) use of computers; and 5) surveillance infrastructure. Indeed, the document checks and visual observations at the facilities revealed that case definitions were posted or easily accessible at $48(96 \%)$ of the health facilities, though lists of immediately and mandatory reportable diseases were only observed in 27 (54\%) of facilities. Graphs of data trends and rumor logs were only available in $11(22 \%)$ and 8 (16\%) of facilities, respectively.

\section{Barriers to Implementation and Suggestions for Improvement}


Thirty-six (72\%) graduates reported encountering obstacles in carrying out surveillance activities after their training. The largest perceived obstacle was a lack of transportation, either because there was no vehicle or no fuel. Other problems included lack of personnel at the district health office, either because positions were not filled or because staff were away at trainings or other activities. Indecision at other levels of the surveillance system and a lack of information technology equipment were also cited as barriers. None of the graduates reported that either they or their colleagues were unfamiliar with surveillance concepts.

All graduates reported being satisfied with the training and with their current skills. They were concerned that if not provided with additional training, they would lose the momentum they had in the first year after completing the program. Several graduates asked for continuing education, and many of them were interested in being part of the FETP Intermediate cohort, a nine-month program also implemented in Guinea. Some graduates recommended enhancing the computer skills portion of the training, and many of them wanted continuing support and supervision from the program.

All supervisors suggested that the graduates would benefit from additional training on analysis software and project management, and that training like that provided by FETP-Frontline would also be of benefit to other employees of the health district.

\section{Discussion}

The evaluation of the first two cohorts of Guinea's FETP-Frontline program provides opportunities to make recommendations to improve the program as well as the evaluation process itself for future cohorts. While identified in the specific context of the Guinean program, some of these recommendations may also be applicable to other similar contexts, in West Africa and beyond.

Based on the demographic characteristics of the first two FETP-Frontline cohorts in Guinea, the evaluation revealed important future considerations for the recruitment of younger, and especially female, individuals to perform key surveillance functions in Guinea. Both initial cohorts were predominantly male $(80 \%)$. One reason behind this disparity is that, because FETP-Frontline is intended to strengthen district surveillance systems, selection for the first two Frontline cohorts in Guinea focused on individuals serving as the District Head of Disease Surveillance and Control. Even though, according to the National Plan for Health Development (2015-2024),[12] women are equally represented in the overall health workforce (51\% female to $49 \%$ male), the senior levels within the Ministry of Health, including in the districts, are primarily male. In this way, gender equality within FETP-Frontline trainees in Guinea is linked to underlying gender disparities within the Ministry of Health, which could be addressed through proactive policies to recruit women to serve as the heads of departments, including Departments of Disease Prevention and Control at the district level.

The relatively high mean age of the first cohort as compared to the second was part of a strategy to ensure the understanding and endorsement of the program from district health office leadership. In line with the strategy, subsequent cohorts, including the second cohort, targeted additional junior staff, 
responsible for the everyday surveillance and field epidemiology field work. Recruitment of junior staff is important to counterbalance the large proportion of the public health workforce in Guinea that will reach the mandatory age of retirement in the next five years. The Ministry of Health needs to prioritize provision of key skills to younger personnel, and particularly women, to galvanize the surveillance workforce and increase gender equity; these efforts may require close coordination with the higher education sector, to ensure a robust educational pipeline that specifically encourages graduates to pursue a career in the public sector. These challenges are not unique to Guinea; between 2013-2017, the regional two-year West Africa Field Epidemiology and Laboratory Training Program trained 48 individuals from 11 countries, but only eight of the graduates were women (17\%). The evaluation report noted that this disparity reflects underlying imbalances in the professionals from which trainees were recruited.[13]

The evaluation revealed that FETP-Frontline graduates in Guinea were acutely aware of the skills they had gained from the training and were able to identify when and how to carry out local-level disease surveillance tasks, including not only collecting data and reporting it through identified channels, but also analyzing data and making recommendations for improvements to stakeholders. Findings indicate that graduates from both cohorts felt their skills had improved since participating in FETP-Frontline. Moreover, tangible outputs from FETP-Frontline graduates at the workplace were observed both by their supervisors and in the health facilities in which they served. For example, all but one of the health facilities had case definitions readily available. These findings mirror outcomes observed in other countries, as reported in the limited available published literature. In Côte d'Ivoire, for example, as in Guinea, the work of graduates of the Surveillance Training for Ebola Preparedness (STEP) program, which has similarities to FETPFrontline in curriculum content, improved the understanding of case definitions and the quality of case investigations.[14]

However, the health facility observations did reveal some gaps. Only just over half (54\%) of the visited health facilities had lists of immediately or mandatory notifiable diseases visibly posted or readily available. It is possible that the absence of these guidelines from other facilities is due to the lists having been recently updated at the time of the evaluation, resulting from Guinea's efforts to review, update, and enhance implementation of its national IDSR framework. However, in 2017, updated IDSR guidance, including lists of reportable diseases and their notification frequency, were distributed to all health facilities in the country. Given the newness of the guidelines, it is particularly important that they should be visibly displayed in all health facilities, to ensure staff are familiarized with the requirements. Similarly, very few $(16 \%)$ of the facilities had a rumor log for suspected cases and outbreaks, and indeed most of the graduates seemed to be unfamiliar with the concept of a rumor log. Some even reported that it did not seem necessary when they had access to verifiable data to use for reporting. With event-based surveillance considered an important component for IHR compliance, and also incorporated into IDSR indicators, it will be important to better integrate these other aspects of surveillance within the FETP training structure. In that way, the planned expansion of FETP in Guinea and the subsequent the increase of FETP graduates working at all levels of the public health system will serve to address some of these observed deficiencies, as decision-makers have a better understanding of the tools required for a robust surveillance system.

Page $14 / 22$ 
A limitation of the evaluation was that it did not directly seek to measure impacts on the surveillance system. However, there is some evidence to suggest a positive benefit of the first two FETP-Frontline cohorts on the surveillance system in Guinea. Data collected during the training showed that the number of facilities integrated into the national surveillance system increased from 450 to 635 . As is the case in many countries, the Ministry of Health in Guinea has limited control and oversight of private sector health facilities, and improving coordination with private for- and not-for-profit health providers is highlighted as a priority in the National Plan for Health Development 2015-2024.[12] To this end, eight FETP-Frontline graduates in Guinea reported improved integration of private clinics into the national surveillance system as a product of the program. As an example, it was a private clinic, added to the surveillance system through the first FETP-Frontline cohort, which provided the $\mathrm{MOH}$ with sufficient data on the 2017 measles outbreak to allow for a formal request for WHO assistance, accelerating the response effort.

There are also data to support improved performance of the surveillance system, although these improvements cannot be directly attributed to the FETP-Frontline training. Timeliness of reporting, for example, increased from $68 \%$ around the start of the FETP-Frontline program to $98 \%$ by the time of the evaluation; the graduates themselves also perceived similar benefits. When the interview team asked graduates to identify the most important outcomes FETP-Frontline had had on the surveillance system, $70 \%$ reported that the quality, completeness, and timeliness of data had improved. This aligns with similar increases observed in other countries; for example, in Benin, average timeliness of surveillance increased almost $130 \%$ after the completion of the first cohort, from $37 \%$ to $85 \%$ in 12 weeks.[5] These data, coupled with the observations regarding the inclusion of private clinics, suggest that future evaluations could benefit from incorporating more direct measures of benefit, as well as impact, on the surveillance system, to further demonstrate the value provided by the FETP-Frontline training. Moreover, further investigations into the mechanisms and approaches used by the FETP-Frontline graduates to incentivize the participation of the private clinics could serve as useful models for other countries' FETP and FETP-Frontline programs.

Another limitation of this evaluation of Guinea's first two FETP-Frontline cohorts was that graduates were asked to score their pre- and post-training knowledge retrospectively, which could have resulted in recall bias. The extent of the recall bias could have been affected by the length of time after completion of the training that the questionnaires were completed, which differed between the two cohorts evaluated. Defining a set timeline for administering pre- and post-training questionnaires, and completing the posttraining evaluation at a standard/routine interval after completion of the program, could reduce potential recall bias; indeed, $C D C$ guidelines recommend conducting the evaluation no more than six months after the conclusion of the training, which was exceeded in the present evaluation. The possibility of desirability bias also cannot be discounted, whereby the respondents consciously or sub-consciously provided responses that would demonstrate the value of the program. The decision not to record the interviews might have helped mitigate some of this bias, as well as encourage the trainees to speak more freely, but reliance on note-taking by the interviewers might have resulted in some lost details or nuances in the recorded responses. The interview questions should also be reviewed by a survey design expert, to 
minimize ambiguity, leading questions, or other sources of inaccuracy, although the questionnaire was field-tested prior to deployment in the evaluation.

Given the successful implementation of FETP-Frontline across many countries since 2015,[5,15] there may be an opportunity to develop a standardized monitoring and evaluation "toolkit", consisting of suggested monitoring indicators aligned with IDSR, template evaluation questions, and other materials designed specifically for FETP-Frontline, which also incorporates standardized direct measures, such as the number of structures contributing to the surveillance system, as well as other indicators that could seek to examine the direct impacts on the FETP-Frontline program on the performance of the surveillance system. To our knowledge, examination of direct impacts is a frequent omission in the evaluation of FETP and other related epidemiological workforce training programs, where the emphasis of evaluation is focused on pre- and post-training knowledge and competencies, participation in surveillance and response activities, and career progression of graduates.[16-20] To this end, a monitoring and evaluation toolkit could still have the flexibility to be customized to fit the specific needs and context of each country, but would standardize data collection between programs and allow for analysis of the benefits and impact of FETP-Frontline on a global, as well as national and regional, scale.

\section{Conclusions}

The evaluation demonstrated a strongly positive perceived benefit of the FETP-Frontline training on the professional activities of graduates in support of surveillance and response functions, as well as the overall surveillance system. However, the demographic analysis of the first two cohorts revealed a substantial gender bias, and a tendency towards recruitment of more senior trainees approaching mandatory retirement age. Given how age and gender are aligned with seniority within the $\mathrm{MoH}$, future recruitment efforts should seek to expand eligibility criteria to ensure more female and early-/mid-career trainees. Gaps were observed regarding the availability of notifiable disease lists, data analysis and trend reports, and especially rumor logs. Given the importance of these tools for accurate and timely reporting of emerging disease threats, in line with Guinea's revised IDSR guidelines and IHR compliance requirements, future FETP-Frontline training should seek to improve availability and use of these instruments. Finally, although improvements to the surveillance system were observed concurrent with the completion of the two cohorts, the evaluation was not designed to directly measure impact on surveillance or response functions. Addressing this aspect of evaluation is critical for establishing an evidence-base to demonstrate the value-added of these epidemiological training programs, and in this way, be used to secure continued political and financial investment in workforce development to advance health security. Combined with the rapid implementation of FETP-Frontline around the world, this suggests an opportunity to develop standardized evaluation toolkits, which could incorporate metrics that would directly assess the benefit and impact of equitable field epidemiology workforce development on countries' abilities to prevent, detect, and respond to public health threats, as well as alignment of desired competencies resulting from the training with other aspects of public health preparedness, response and resilience.[21] The recent establishment of new regional bodies, such as the Africa Centres for Disease 
Control and Prevention Institute for Workforce Development, present an important opportunity for leading and consolidating such efforts in the future.[22]

\section{Declarations}

\section{Ethics approval and consent to participate}

The study was determined to be exempt from IRB approval as primary data collection was conducted via interviews with individuals responding in their official capacity as trainees of a government program. The Ministry of Health provided permission to conduct the evaluation.

\section{Consent for publication}

Not applicable.

\section{Availability of data and materials}

The datasets used and analyzed during the current study are available from the corresponding author on reasonable request.

\section{Competing interests}

The authors declare no competing interests.

\section{Funding}

This publication was supported by Cooperative Agreement U19GH001591 funded by the U.S. Centers for Disease Control and Prevention. Its contents are solely the responsibility of the authors and do not necessarily represent the official views of the Centers for Disease Control and Prevention or the Department of Health and Human Services.

\section{Authors' contributions}

DC revised the questionnaires, provided interview and coding training, collected the data, helped with data entry, performed the data quality control, contributed to the analysis, supervised the coding of the data, and wrote the first drafts of all sections. MBB and MB also provided training, collected data, and managed the logistics of the data collection activities; BID provided project oversight and obtained permissions from the MoH. SC contributed to data collection. CJS and LDM contributed to the writing and revision of drafts. LC contributed to adaptation of the questionnaires and feedback on the initial 
evaluation report. PDMM contributed to the conceptualization of the evaluation and the methods and reviewed all drafts. All authors reviewed and approved the final manuscript.

\section{Acknowledgements}

We wish to acknowledge the efforts of all the Epi-Detecte field epidemiologists and staff who spent weeks in the field conducting interviews and collating the questionnaires. We also thank Mamadou Kadiatou Diallo, the GIS specialist who developed the maps and acted as the lead data manager. We are grateful for the support of the Guinea Ministry of Health, including Dr. Facinet Yattara, who generously gave permission for the evaluation, and to all the trainees, their supervisors and health facility staff who gave their time to complete the interviews.

\section{References}

1. The World Bank. Implementation Completion and Results Report (TF-14785). Washington; 2018.

2. Afriyie DO, Nyoni J, Ahmat A. The state of strategic plans for the health workforce in Africa. BMJ Glob Health BMJ Specialist Journals. 2019;4:e001115.

3. Baize S, Pannetier D, Oestereich L, Rieger T, Koivogui L, Magassouba N, et al. Emergence of Zaire Ebola Virus Disease in Guinea. N Engl J Med Massachusetts Medical Society. 2014;371:1418-25.

4. Dahl BA, Kinzer MH, Raghunathan PL, Christie A, De Cock KM, Mahoney F, et al. CDC's Response to the 2014-2016 Ebola Epidemic - Guinea, Liberia, and Sierra Leone. MMWR Suppl. 2016;65:12-20.

5. André AM, Lopez A, Perkins S, Lambert S, Chace L, Noudeke N, et al. Frontline Field Epidemiology Training Programs as a Strategy to Improve Disease Surveillance and Response. Emerg Infect Dis. 2017;23:166-73.

6. Bhatnagar T, Gupte MD, Hutin YJ, Kaur P, Kumaraswami V, Manickam P, et al. Seven years of the field epidemiology training programme (FETP) at Chennai, Tamil Nadu, India: an internal evaluation. Hum Resour Health. 2012;10:36.

7. López A, Cáceres VM. Central America Field Epidemiology Training Program (CA FETP): a pathway to sustainable public health capacity development. Hum Resour Health. 2008;6:27.

8. CDC. Multisite Evaluation of Field Epidemiology Training Programs. Findings and Recommendations. Atlanta; 2014.

9. Schneider D, Evering-Watley M, Walke H, Bloland PB. Training the Global Public Health Workforce Through Applied Epidemiology Training Programs: CDC's Experience, 1951-2011. Public Health Rev Springer Science Business Media LLC. 2011;33:190-203.

10. Mmbuji P, Mukanga D, Mghamba J, Ahly M, Mosha F, Azima S, et al. The Tanzania Field Epidemiology and Laboratory Training Program: building and transforming the public health workforce. Pan Afr Med J. 2011;10:9. 
11. Roka ZG, Githuku J, Obonyo M, Boru W, Galgalo T, Amwayi S, et al. Strengthening health systems in Africa: A case study of the Kenya field epidemiology training program for local frontline health workers. Public Health Rev. BioMed Central Ltd.; 2017;38.

12. Ministère de la Santé. Plan National de Développement Sanitaire (PNDS) 2015-2024. Conakry; 2015.

13. The World Bank. IMPLEMENTATION COMPLETION AND RESULTS REPORT (TF-14785) ON A GRANT IN THE AMOUNT OF US\$ 10 MILLION TO THE Economic Community of West African States. - West Africa Health Organization (ECOWAS-WAHO) FOR A West Africa Regional Disease Surveillance Capacity Strengthening Project (WARDS). 2018 Jan.

14. Cáceres VM, Sidibe $S$, Andre M, Traicoff D, Lambert S, King M, et al. Surveillance Training for Ebola Preparedness in Côte d'Ivoire, Guinea-Bissau, Senegal, and Mali. Emerg Infect Dis. 2017;23:174-82.

15. Establishing TEPHINET. FETP Frontline Across Latin America and the Caribbean in Response to Zika I TEPHINET [Internet]. [cited 2020 Jan 21]. Available from: https://www.tephinet.org/establishingfetp-frontline-across-latin-america-and-the-caribbean-in-response-to-zika.

16. Lau CQ, Johnson E, Amaya A, LeBaron P, Sanders H. High Stakes, Low Resources: What Mode(s) Should Youth Employment Training Programs Use to Track Alumni? Evidence From South Africa. J Int Dev. 2018;30:1166-85.

17. Gatei W, Galgalo T, Abade A, Henderson A, Rayfield M, McAlister D, et al. Field Epidemiology and Laboratory Training Program, Where Is the L-Track? Front Public Health. 2018;6.

18. Lescano AG, Salmon-Mulanovich G, Pedroni E, Blazes DL. Epidemiology: Outbreak investigation and response training. Science. 2007. p. 574-5.

19. Jones D, Caceres V, Herrera-Guibert D. A tool for quality improvement of field epidemiology training programs: Experience with a new scorecard approach. J Public Health Epidemiol. 2013;5:385-90.

20. TEPHINET. Accreditation I TEPHINET [Internet]. [cited 2020 Jan 21]. Available from: https://www.tephinet.org/accreditation.

21. Olu O, Usman A, Kalambay K, Anyangwe S, Voyi K, Orach CG, et al. What should the African health workforce know about disasters? Proposed competencies for strengthening public health disaster risk management education in Africa. BMC Med Educ. 2018;18:60.

22. Africa CDC. Institute for Workforce Development [Internet]. Afr. CDC. [cited 2021 Nov 18]. Available from: https://africacdc.org/institutes/africa-cdc-institute-for-workforce-development/.

\section{Figures}




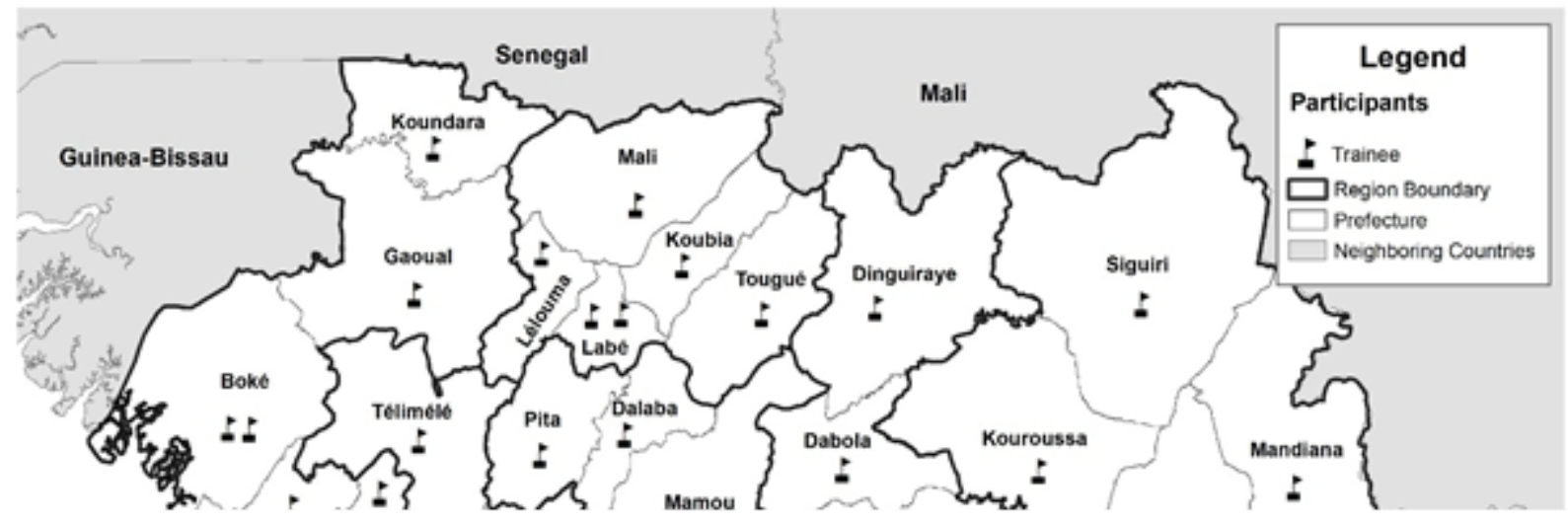

Figure 1

Map of Districts Staffed by Guinea FETP-Frontline Graduates, Cohorts 1 and 2 


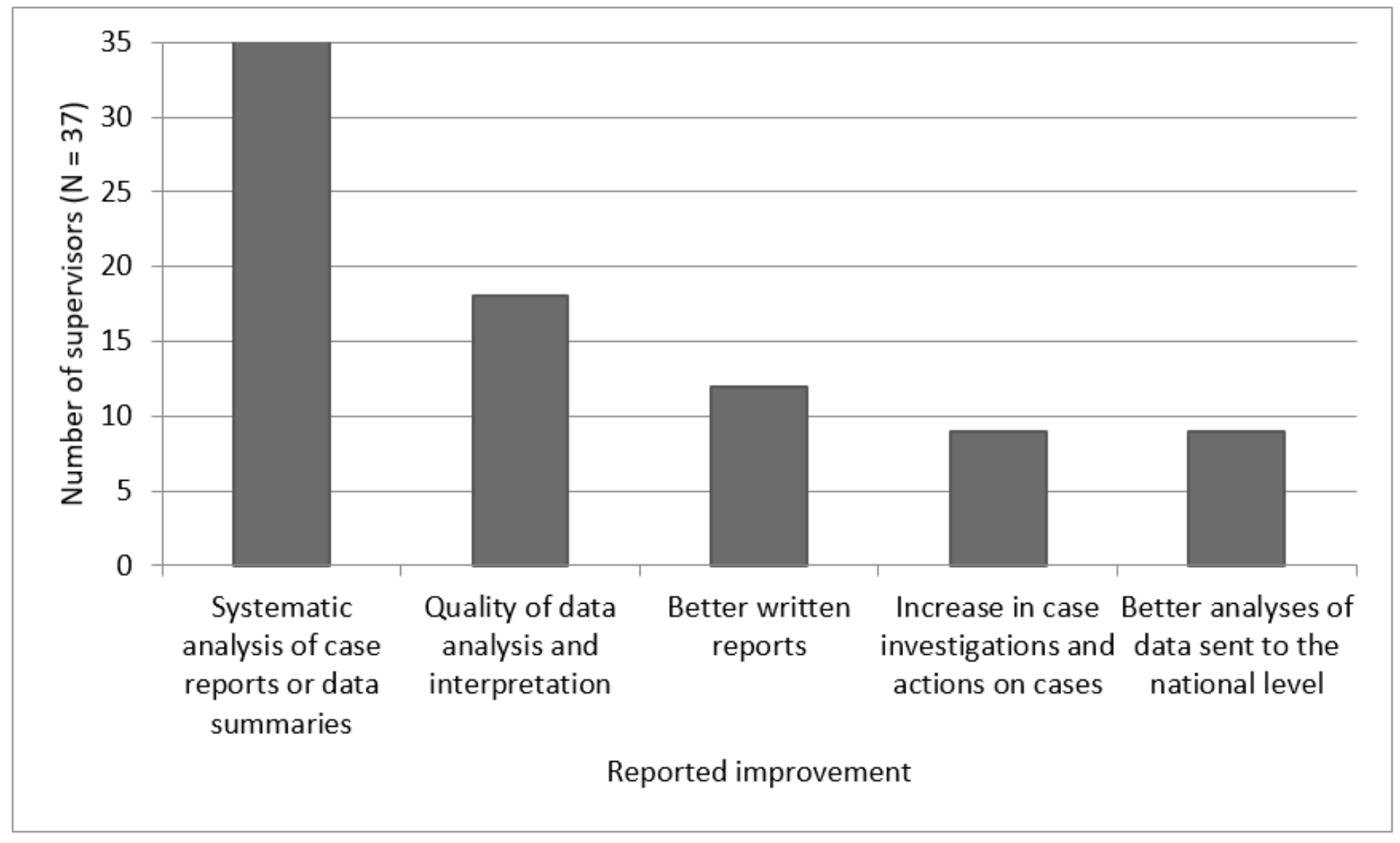

\section{Figure 2}

Summary of improvements reported by supervisors for FETP-Frontline program graduates 


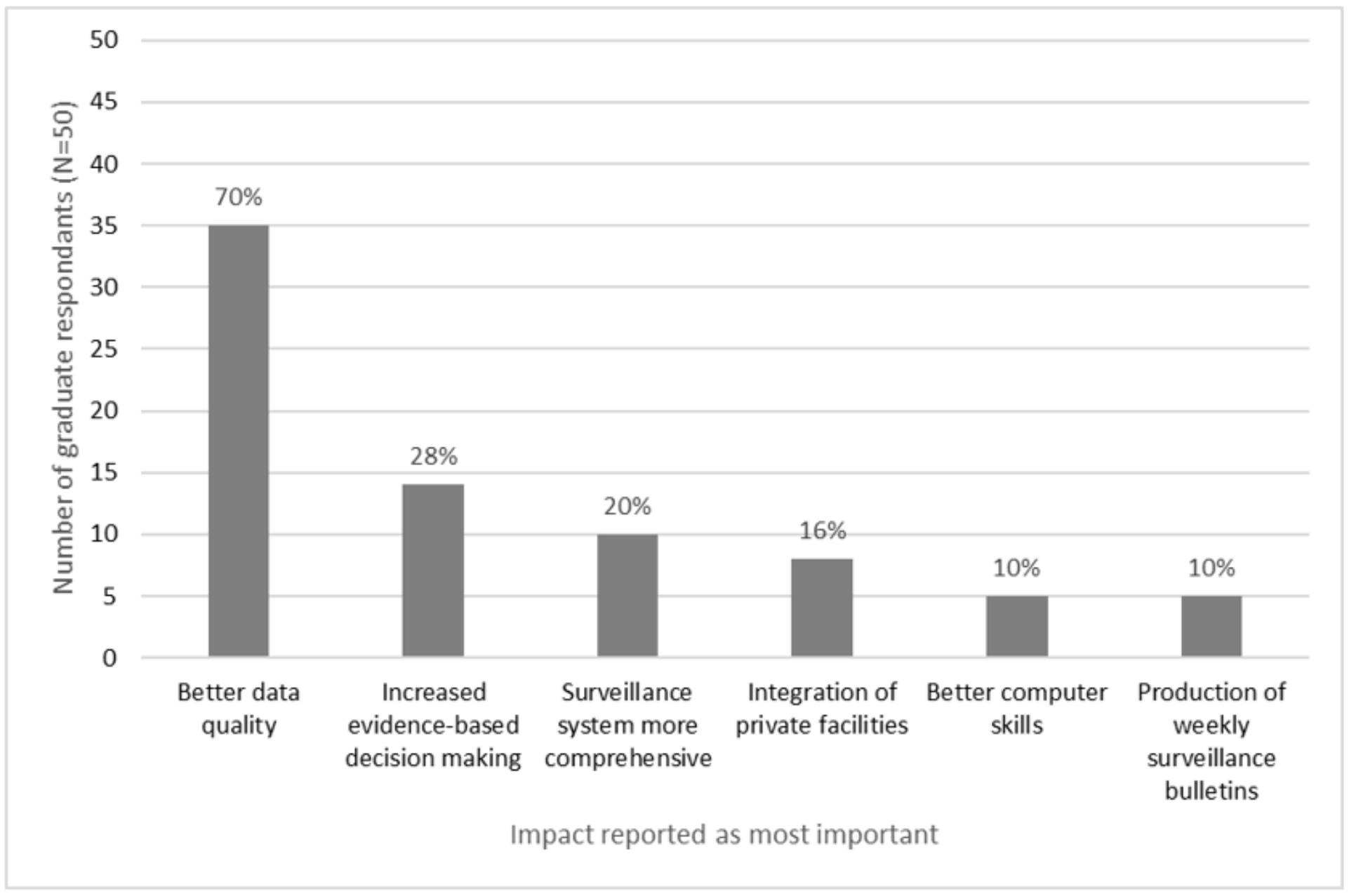

Figure 3

Graduate perceptions on the most important outcome of FETP-Frontline training on the surveillance system 